\title{
La influencia de la oralidad en las canciones de Xabier Lete*
}

\author{
The Influence of Orality in the Songs \\ of Xabier Lete
}

Jon MARTIN-ETXEBESTE

Universidad del País Vasco

RECIBIDO: 9 DE MARZO DE 2020 Campus de Bizkaia, Sarriena auzoa. Leioa, 48940 jon.martine@ehu.eus

Orcid ID 0000-0002-0137-9150

Resumen: En este trabajo se analizan las evidencias de oralidad en las canciones de Xabier Lete (1944-2010). Este cantautor fue uno de los mejores poetas vascos del siglo XX. La tradición vasca del bertsolarismo influyó directamente en muchas de sus canciones que destacan por tener una notable influencia de la oralidad. Esta tendencia puede observarse tanto en la métrica como en el estilo. En este estudio se clasifican sus canciones según el grado de oralidad, se repasa la evolución de su discografía, y se analizan tres de sus canciones para profundizar en los rasgos orales que ofrecen.

Palabras clave: Xabier Lete. Oralidad. Bertsolarismo. Textos transicionales. Ez Dok Amairu.

\begin{abstract}
This work analyses the songs of Xabier Lete (1944-2010) to detect characteristic features of orality. Lete was one of the best Basque poets of the $20^{\text {th }}$ century, and his lyrics were influenced by the Basque tradition of bertsolarismo. This tendency can be seen in both the metrics and the style. This study classifies his songs according to their oral influence, observes the evolution of his discography, and analyses three of his songs in search of features of orality.
\end{abstract}

Keywords: Xabier Lete. Orality. Bersolaritsmo. Transitional Texts. Ez Dok Amairu.

\footnotetext{
* Este artículo forma parte del proyecto del Grupo Consolidado de Investigación LAIDA (Literatura eta Identitatea) que pertenece a la red de Grupos de Investigación del Gobierno Vasco (IT 1397/19) y está reconocido por la Universidad del País Vasco/Euskal Herriko Unibertsitatea (PPGA 20/19).
} 


\section{INTRODUCCIÓN}

a tardía incorporación a la letra impresa ha convertido la literatura escrita en euskara en una rara avis. Su tradición está fuertemente marcada por la oralidad. Conocer las reglas del bertsolarismo es fundamental para entender la obra de algunos poetas, ya que muchos de sus poemas son transicionales. ${ }^{1}$ Este trabajo se centra en Xabier Lete (Oiartzun, 1944-2010).

Este cantautor estaba fascinado por los bertsolaris de la época de su infancia y el gusto por esta disciplina está plasmado en su obra. Su afición por la literatura afloró en su niñez, gracias a que tuvo acceso a multitud de libros a través de su tío, que era cura. A los 13 años, cuando estudiaba en la academia La Magdalena de Errenteria-Orereta, se topó con Ricardo Moreno, con quien se aficionó a la literatura. Durante los años en los que estudió en la Universidad Laboral de Tarragona, se cultivó tanto en la literatura como en el cine. Volvió a su pueblo natal transformado en una persona con inquietudes culturales y fue acogido por la familia Lekuona, una saga de intelectuales que le instruyeron tanto en el ámbito de la literatura universal como en el de la popular.

Cuando, en 1965, se incorporó al colectivo Ez Dok Amairu, Lete era un escritor novel que empezaba a destacar por su poesía y sus artículos de opinión. También estaba ligado al mundo del teatro.

Ez Dok Amairu fue un colectivo de músicos y escritores que, como muchos otros grupos de cantautores, floreció a finales de los años 60 y criticó duramente la represión imperante. El colectivo fue vanguardista y cumplió una función muy importante para el País Vasco. El grupo tuvo una gran importancia como divulgador del patrimonio oral vasco. En pleno franquismo y bajo una implacable dictadura los artistas compaginaron obras de su autoría con poemas, baladas y textos que habían recopilado ellos mismos u otros investigadores (como Resurrección María de Azkue). Lete escribía en 1977 (23) que los poetas del grupo tuvieron que modular sus letras para que pasaran la censura y esto contribuyó a un estilo metafórico y sombrío.

Aunque comenzó su andadura como letrista, al igual que Jose Anjel Irigaray y Joxan Artze, al poco tiempo dio el salto a la música y se convirtió en cantautor comparable, en una escala vasca, a autores como Paco Ibáñez o Jacques Brel. Estableció una gran conexión con el público, ya que era un gran comunicador.

1. Ver Barandiaran para un estudio de un aspecto actual de la cuestión, y Gintsburg para un fenómeno paralelo en el Magreb. 
Estuvo casado con Lourdes Iriondo (1935-2005), que también formaba parte del colectivo, y era la artista con más proyección de la época. Junto a ella publicó varios discos. Lete también trabajó en solitario, escribiendo nuevas canciones, traduciendo canciones de artistas contemporáneos, y rescatando textos de años atrás. Fue también un gran poeta que publicó numerosos libros. En 2009, su libro Egunsentiaren esku izoztuak (Las ateridas manos del alba) recibió el Premio Euskadi de Literatura.

La influencia de la oralidad en Lete es evidente, sobre todo en sus canciones. El bertsolarismo (bertsolaritza en euskera) es probablemente su mayor influencia. El bertsolarismo es una disciplina de improvisación oral cantada en la cual la persona que canta lo hace sobre una tonada que le obliga a respetar una métrica y una rima. Los bertsos, estrofas, son creados frente a un público, y normalmente responden a lo que acaba de cantar otro bertsolari. Actualmente la disciplina que más éxito tiene es la improvisatoria, pero durante algunos años los bertsos escritos cobraron mucha importancia, ya que eran un medio de comunicación de ideas muy efectivo. El bertsolarismo data de, al menos, el siglo XV aunque, evidentemente, ha cambiado mucho durante la historia. Hoy en día se celebran campeonatos que reúnen presencialmente a más de 14000 personas, y los mejores bertsolaris son reputados artistas.

En lo que se refiere a aspectos formales, los bertsolaris deben respetar una métrica concreta y lo hacen improvisando sobre una tonada. Para ello, suelen elegir entre las 3000 tonadas cuál es la que mejor se adapta al tema propuesto y al enfoque que le quiere dar. Una gran parte de esas tonadas son de ocho versos y, dependiendo del número de sílabas, pueden ser zortziko mayor (versos impares de 10 sílabas y pares de 8) o zortziko menor (7 y 6 sílabas) (Garzia/ Sarasua/Egaña 88-89).

El bertsolarismo tiene una gran importancia en el País Vasco. Es una disciplina centenaria, que ha perdurado por la tardía incorporación a la literatura escrita en euskera y las prohibiciones que ha tenido este pueblo. En esta disciplina prima la improvisación y su creación, por ser tan efímera, ha desaparecido en gran parte. Se conservan algunos bertsos gracias a la memoria colectiva y porque en algún punto de la Historia alguien los plasmó en un papel. En cambio, se conservan muchos bertsos escritos y estos han servido tanto para conservar tanto otra versión de la Historia, como para definir un estilo de lenguaje oral.

Oiartzun, el pueblo que vio crecer a Lete, era la cuna del bertsolarismo. El mejor bertsolari de la década de los 60, Manuel Olaizola "Uztapide", vivía en 
su mismo pueblo; y otro de los mejores, Jose Joakin Mitxelena, también había nacido en Oiartzun. Cualquier actuación de bertsolaris era seguida con devoción por parte del público. Lete fue un apasionado del bertsolarismo (Lete 2011, 55), y -aunque no se ha recalcado lo suficiente- uno de los mejores escritores de bertsos de su tiempo junto, a Xalbador y Amuriza (Aulestia 180). En sus primeras actuaciones, eligió cantar bertsos escritos por Txirrita, y fueron del agrado del público. Después, publicó varios discos monográficos. El trabajo más destacable data del año 2001 y consta de siete discos que recogen la obra más relevante de diez bertsolaris del siglo XX. Su pasión proviene de una edad temprana en la que los bertsolaris más importantes visitaban Oiartzun. En una entrevista relata que hablaban con gran autoridad y el público les escuchaba con gran atención (Lete 2011, 25).

\section{EL CORPUS}

Para hacer el estudio de las letras de las canciones de Lete se ha tenido que hacer una selección. Las canciones que publicó son, en total, 116. Siendo el criterio de selección la investigación de los procesos creativos de Lete, se han descartado las canciones traducidas de otros autores. También han quedado fuera del corpus casi 70 canciones que llevan sus letras, pero cantadas por otros artistas. Evidentemente, para no crear una distorsión, también se han descartado los siete discos que Lete dedicó al bertsolarismo. En ellos recopila decenas de tonadas con estructura métrica del bertsolarismo. Estos bertsos pudieron haber constituido un humus creativo de nuevos poemas, pero como no son de su autoría, no se han incluido en el análisis. Con estos parámetros, el corpus lo componen un total de 39 canciones. En ellas el autor pudo expresarse libremente, usando las palabras más apropiadas para convertirse en canción (y que, a su vez, esquivaran la censura). Hay varias canciones que Lete grabó dos e incluso tres veces, y en muchas ocasiones introducía un nuevo matiz. En esos casos la letra que se ha tenido como referencia ha sido la que aparece en Abestitzak eta poema kantatuak, obra en la que Lete reescribe y da por acabada su labor de reescritura.

\section{ANÁLISIS DE LA MÉTRICA}

En este trabajo se han buscado señas de identidad de los textos orales en las canciones de Lete. Una de las señas más objetivas es la métrica. Hay patrones tradicionales del bertsolarismo que pueden ser identificados fácilmente. El ber- 
tsolarismo usa decenas de estructuras métricas, pero hay dos que son especialmente recurrentes: las tonadas menores combinan versos de 7 y 6 sílabas; la rima se ubica en el segundo verso (el primer verso está libre de rima). Las tonadas mayores, en cambio, constan de versos de 10 y 8 sílabas; en esta métrica también es el verso par el que rima (Garzia 88). La mayoría de las tonadas recurren a esa combinación y la repiten dos, tres, cuatro o cinco veces. Para mostrar un ejemplo se utilizará la canción "Seaska kanta" (Canción de cuna), separando las sílabas y subrayando las rimas:

7 Haun-di-tzen ze-ra-ne-an

6 i-ku-si-ko du-zu

7 i-si-lik e-go-te-ak

6 zen-bat ba-li-o dun.

7 Zo-az zu-re bi-de-tik

6 ho-be da ho-rre-la

7 gau-za de-nen gai-ne-tik

6 hai-ze-a be-ze-la. (Lete 2006, 49)
Cuando te hagas mayor

observarás

que valioso es mantenerse callado.

Sigue tu camino, será lo mejor, por encima de todo como el viento.

Esta estructura es un zortziko txikia (zortziko menor). La palabra zortziko (de ocho) señala el número de versos (ocho) y txikia (menor) se refiere a la combinación de sílabas: en este caso, 7 y 6 .

La estructura a la que más recurre el autor es el zortziko mayor. Sirve, como ejemplo, este fragmento de "Haizea dator ifarraldetik" (El viento viene del norte) de Lete, en el que se señala la rima y se ha separado por sílabas:

10 I-tzal- gaiz-to-ak i-sur-tzen di-ra

8 men-di he-ga-le-tan behe-ra

10 ar-gi a-pa-lak ba-do-az lais-ter

8 bat-ba-te-an i-tzal-tze-ra.

10 i-zen ze-ha-tzak uz-ten di-tu-gu

8 e-ror-tzen zu-lo bel-tze-ra

10 pres-ta-tzen ge-ra e-txe hus-tu-a

8 a-rrotz guz-ti-ei sal-tze-ra. (Lete 2006, 87)
Las malvadas sombras se vierten sobre las laderas del monte; las débiles luces huyen, y se apagan súbitamente. Dejamos que nombres concretos a un oscuro agujero caigan $\mathrm{y}$ nos disponemos a vender a un extraño nuestra casa vacía. ${ }^{2}$

Según veremos en la tabla que sigue, la mayoría de las canciones de Lete concuerdan de alguna manera con las estructuras antes mencionadas. Algunas de

2. Todas las traducciones son del autor del artículo. 
estas canciones han sido adoptadas por el bertsolarismo y se han convertido en tonadas que se usan habitualmente para improvisar; por ejemplo, "Habanera", "Neguaren zai" ('Esperando al invierno'), "Haizea dator ifarraldetik" o "Izarren hautsa" ('Polvo estelar').

Otras, en cambio, no han sido adoptadas para la improvisación, aunque métricamente fueran propicias: Por ejemplo, "Lore gorrien balada" ('La balada de las flores rojas'), "Euskalerri nerea" ('País Vasco mío'), "Maiteaz galdezka" ('Preguntando por mi amada') o "Ez dut amets handirik" ('No poseo grandes sueños'). Quizás porque la melodía no fuera del gusto de los bertsolaris, por desconocimiento, o simplemente por falta de iniciativa.

En otros casos, algunas de las canciones tienen versos de 6, 7, 8 o 10 sílabas, pero no siguen un patrón típico (contienen versos libres o el orden de los versos es atípico).

En último lugar, hay un grupo de canciones que nada o poco tiene que ver con la métrica del bertsolarismo:

\begin{tabular}{l|c} 
Tonadas que se usan comúnmente en el bertsolarismo & 10 \\
\hline $\begin{array}{l}\text { Tonadas con métricas propicias para la improvisación } \\
\text { pero no usadas para tal propósito }\end{array}$ & 9 \\
\hline Puede tener relación con el bertsolarismo & 8 \\
\hline Rara o nula relación & 12 \\
\hline
\end{tabular}

Tabla 1. Relación de las canciones con la métrica del bertsolarismo.

Aunque no sea exacta, esta clasificación sirve para señalar la influencia del bertsolarismo en las canciones de Lete. Se puede afirmar que 27 de las 39 canciones están ligadas a la tradición, métricamente; y al menos 19 se corresponden exactamente. Cabe apuntar que alguna de las tonadas que compuso Lete no correspondía a la tradición vasca, sino a la española (puramente octosílaba); pero a raíz de la creación de tal tonada, esta estructura métrica se ha convertido en una de las más comunes para la improvisación en solitario. Es decir, que no solo tendríamos que tener en cuenta la influencia del bertsolarismo en Lete, sino la influencia de Lete en el bertsolarismo. De hecho, Lete es uno de los artistas que más tonadas ha aportado, con más de 40 tonadas creadas según la base de datos Xenpelar. 


\section{Clasificación ORAL}

También se podría hacer otra clasificación que no se guíe tanto por la estructura como por su contenido y se fije en indicios de oralidad. Juan Mari Lekuona, abanderado de la investigación de la literatura oral vasca, la contrapone a la literatura escrita afirmando que surge y perdura al margen de la escritura y pervive gracias a la memoria colectiva. Afirma que la oralidad está ligada a las leyes socioculturales vigentes en estadios anteriores a la literatura escrita, y en cuanto a técnica, las obras se mantienen ligadas a normas originarias ya fijadas $(211,212)$. En este trabajo el concepto de oralidad no se define como la memoria rescatada de autores anónimos, sino que se centra en el estilo de escritura del texto en el que el autor está más interesado en comunicar, ser entendido a la primera, y cuya narración incluso pueda tener cambios bruscos o pequeños defectos fácilmente remediables de forma escrita. Es decir, defiende la espontaneidad de la oralidad como contrapunto a los textos perfectamente planeados.

\section{TeXto TRAnsicional}

Siguiendo las pautas de Sarali Gintsburg (2017, 478a), se definirá como texto de transición el texto creado por un poeta alfabetizado que pretende simular el estilo de la tradición oral, aunque lo haga con la ayuda de la escritura. El reto más interesante será, por tanto, determinar cuáles son estas características orales de los textos transicionales.

En gran parte dependerá de la expresión que se esté analizando. En este caso, se buscan indicios de oralidad (sobre todo influencias del bertsolarismo) en canciones. Estos indicios están reflejados de forma más detallada y con ejemplos en el análisis de tres canciones de Lete. Se pueden adelantar las siguientes características como marcas del lenguaje oral:

- Mensaje principal comprensible en la primera escucha.

- Uso de fórmulas, patrones o guiones identificables. Métricas tradicionales y grupos de rimas frecuentemente usadas.

- Frases construidas de forma no convencional (hipérbaton, ausencia de conjunciones, elipsis...) con el fin de cumplir una métrica o rimar.

- Incorrecciones: léxicas, sintácticas, contracciones, deformaciones lingüísticas, falta de concordancia...

- Obra inacabada: la reescritura. 


\section{REESCRIBIR}

Es interesante ver cómo, con el paso del tiempo, Lete modifica sus letras al igual que muchos cuentacuentos o juglares modificarían sus piezas, reformulándolas. Ese sería, por ejemplo, el caso de una de sus canciones más famosas: "Habanera", la cual está anteriormente publicada por partes como poemas, y reescrita para convertirla en canción. También modeló muchas de sus canciones para matizarlas. En su primer EP canta la canción "Maiteaz galdezka" de la siguiente forma:

Bakardade haundi bat neukan bihotzean, negar egiten nuen zutaz oroitzean.

Galdera egin nuen etsipen hotzean, gizonok nora goaz mundutik hiltzean? (Lete 2006, 54)
La soledad asolaba mi corazón lloraba cuando me acordaba de ti. Pregunté en mi fría desolación: ¿Adónde van los hombres cuando mueren?

En una versión posterior, la tercera línea se modifica por "galdera egin nion Jaunari hotzean" ('pregunté fríamente a Dios'). Como se puede observar, esta segunda versión es más específica, ya que sustituye la "fría desolación" que sentía, e interpela directamente a Dios. Este modo de proceder se repite a menudo en las canciones de Lete y es especialmente interesante observar cómo modula su lenguaje bélico dependiendo de la situación política del momento.

\section{FÓRMULAS}

Se usará la definición de fórmula que propuso Milman Parry (77) cuando la definió como grupo de palabras que se usan frecuentemente bajo las mismas condiciones métricas para expresar una idea esencial. En la improvisación de los bertsolaris las fórmulas son algo ineludible. Linda White afirma lo siguiente:

In bertsolarismo we cannot explain the phenomenon in terms of multiformity, where entire lines can be substituted by others in the retelling of an old tale. The tales are contemporary, dealing with modern topics which are assigned moments prior to the performance, and the repetition of lines, indeed, of words, would be judged a fault in Basque bertsoak. (248)

Lete, en cambio, pese a disponer de la ventaja de no tener que improvisar, decide no corregir, mejorar o evitar ciertas fórmulas, sino escribirlas tal y como lo haría una persona que improvisaba. Wray afirma (47) que en una sociedad 
alfabetizada el grado en que un orador utiliza fórmulas está vinculada a varios factores. En este caso Lete intuye que hay una alta probabilidad de que la persona oyente comprenda la fórmula y desea usarla como seña de identidad compartida.

Es el gusto por la fórmula. Es el conocimiento de la tradición el que hace que algunas canciones de Lete parezcan orales. Foley habla de poemas escritos orales $(2002,40)$ que parecen una contradicción en sí pero son muy importantes.

Los poemas escritos orales se disfrutan como si fueran completamente orales. Eso da al oyente la oportunidad de participar en la creación de la canción e intentar adivinar la siguiente frase. Percibió algo similar durante la final nacional de bertsolarismo del 2005:

La interacción entre performador y audiencia es sorprendente. Al ser tan fino el patrón de rima y ritmo, hay veces en las que el horizonte se ilumina, ya que los oyentes pueden adivinar las últimas líneas de un bertso que nunca antes se había cantado; esto es un gran ejemplo de la gran comunión que hay entre público y bertsolari. (Foley 2007, 5)

Según los criterios citados, podemos dividir las canciones de Lete en dos grupos:

- Canciones con notable influencia oral: 15

- Canciones con poca influencia oral: 24

"Euskalerri nerea”, "Maiteaz galdezka", "Seaska kanta", "Ez dut amets handirik", "Gizon arruntaren koplak” ('Coplas de un hombre común'), "Langile baten seme" ('Hijo de un obrero'), "Teologia, ideologia” y "Henry Millerri eskutitza" ('Carta a Henry Miller') son las canciones con más influencia oral en la discografía de Lete. "Zuk badiozu" ('Si tú lo dices'), "Lore gorrien balada" y "Otsoak eta txanogorritxu” ('Caperucita y los lobos') también tienen una influencia notable.

La métrica es, la mayoría de las veces, un indicador fiable del grado de oralidad que se puede esperar de los textos de Lete. De hecho, las canciones recién mencionadas son, en su mayoría, estrofas que coinciden con el bertsolarismo. El hecho de tener que encorsetar las palabras puede crear una estética (ausencia de conjunciones, elipsis...) que evoca cierta oralidad. Pero hay algunas de las canciones que cumplen a rajatabla los patrones métricos del bertsolarismo, pero quedan fuera de este grupo por tener una carga poética difícilmente digerible en una sola escucha. En estos casos el continente y el 
contenido no coinciden. Es el caso de canciones como "Haizea dator ifarraldetik" o "Neguaren zai". Ambas están escritas en la estructura más común (zortziko mayor) pero sus textos no están pensados para ser entendidos en la primera escucha; prima la calidad poética sobre la comunicación:

$\begin{array}{ll}\text { Ez nau izutzen negu hurbilak } & \text { No me asusta el invierno que se acerca } \\ \text { uda beteko beroan } & \text { en el calor del verano } \\ \text { dakidalako irauten duela } & \text { porque sé que el presente } \\ \text { orainak ere geroan } & \text { también perdura en el futuro } \\ \text { izadiaren joan geldian } & \text { en el pausado devenir de la naturaleza } \\ \text { en la línea de generaciones } \\ \text { gizalditako lerroan } & \text { hasta que todo sea presente } \\ \text { nor izanaren erroan. (Lete 2006, 94) } & \text { en la raíz de lo que somos. }\end{array}$

Algunas canciones son claramente poéticas y se alejan de la oralidad, pero hay otras en las que la clasificación no es tan clara. Nueve de las 39 canciones ofrecen muchas dudas. Se pueden encuadrar dentro de una zona mixta, y se podrían clasificar en cualquiera de las posiciones según como se pondere la importancia de cada característica oral. Este trabajo ha optado por clasificar las canciones "Sinisten dut" ('Yo creo'), "Izarren hautsa", "Cançó a Catalunya" ('Canción a Catalunya') y "Gauaren ordezko eguna” ('El día que sustituye a la noche') como predominantemente orales y en cambio "Poeta hoiek" ('Esos poetas'), "Habanera", "Haizea dator ifarraldetik", "Neguaren zai" y "Jardin bat zuretzat" ('Un jardín para ti') como no orales.

Esta clasificación sirve solo para una exploración superficial, para destacar una tendencia. Se ha considerado que tienen una notable influencia oral las canciones que están escritas de manera poco críptica, las que no tienen muchas elipsis, y se centran en contar una idea sin mucha ornamentación. Aun con estas características, la clasificación es subjetiva y discutible.

\section{EVOLUCIÓN DE LO ORAL A LO ESCRITO Y A LA INVERSA}

Lete publicó numerosos discos. Sus primeras publicaciones fueron maquetas con canciones que se afianzaron en discos posteriores. Normalmente combinaba una labor de recuperación del patrimonio cultural vasco con traducciones de autores contemporáneos, tanto nacionales como internacionales, y sus propias creaciones. Las canciones con influencia oral o escrita han tenido un peso diferente en cada disco. Siete de sus discos contienen canciones propias 
y serán las que servirán para la comparativa. El primer EP que publicó Xabier Lete levó su nombre (se nombra como XL1EP en el gráfico). Lete publicó en él cuatro canciones de las cuales tres llevaban su letra. Dos de ellas se pueden considerar orales y así lo refleja el gráfico. Estos son los demás discos publicados: "Segundo EP Xabier Lete" (XL2EP), "Primer LP Xabier Lete" (XL1LP), "Lourdes Iriondo eta Xabier Lete" (LI\&XL), "Kantatzera noazu" (KN), "Lore bat zauri bat" (L1Z1), "Eskaintza" (ESK) y el CD póstumo de su concierto en Errenteria-Orereta (KON). Los discos están ordenados cronológicamente y se han tenido en cuenta las características de los textos orales (uso de fórmulas identificables, comprensibilidad inmediata, etc.) para inclinar la balanza hacia una consideración oral o no oral.
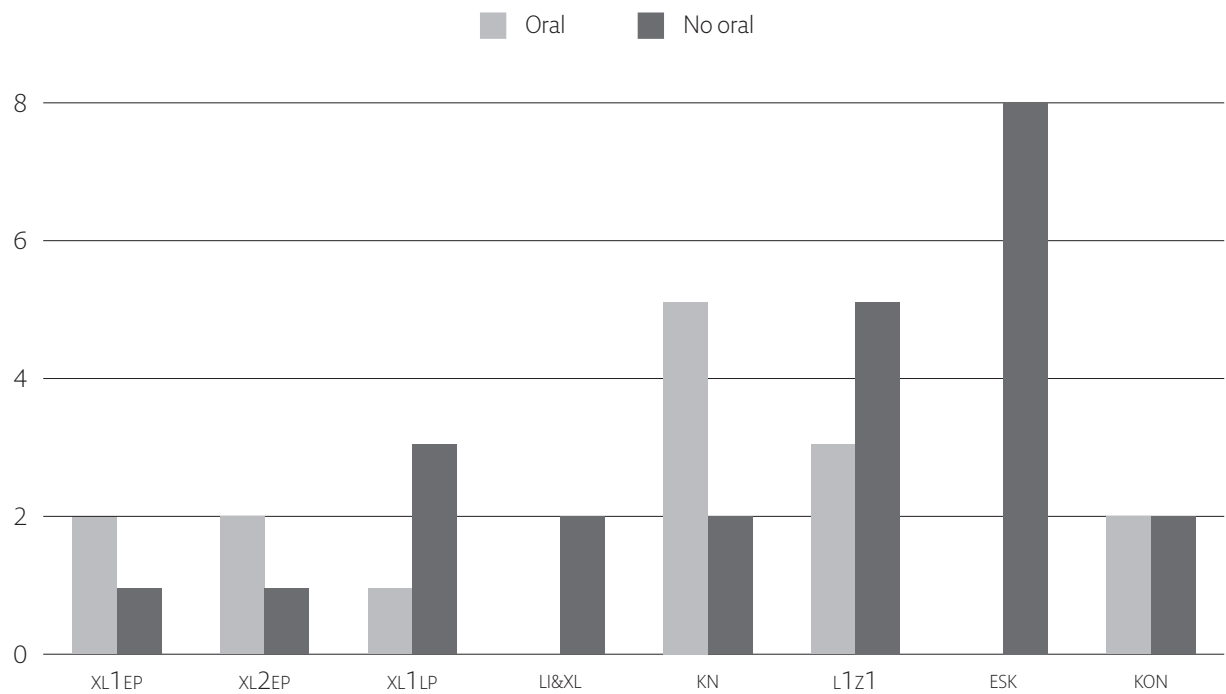

Figura 1. Cantidad de canciones con influencia oral y no oral

Si se analiza la discografía de Lete (tomando solo en cuenta nuevas canciones), pueden observarse algunos cambios de tendencia: en sus dos primeros EPs predominan las canciones con gran influencia del estilo oral. En cambio, en su primer LP esta tendencia se invierte. El cuarto disco que publica junto a Iriondo es más complejo de analizar ya que solo publica dos canciones y ambas son cantadas por ella. Esto las coloca al borde del corpus, ya que se desecharon las canciones escritas por Lete pero cantadas por otras voces. Pero, a su vez, el disco también está firmado por Lete, por lo que finalmente se incluyeron en el corpus. Las dos canciones tienen una baja influencia oral. 
En 1976 llegó "Kantatzera noazu", el disco más oral de su carrera, del cual se analizarán dos canciones: "Langile baten seme" y "Gizon arruntaren koplak".

Solo dos años después publicó "Lore bat zauri bat", en el cual puede verse, claramente, un cambio de tendencia. Lete opta por un estilo en el que el/la oyente tiene que hacer un esfuerzo mayor para adquirir todos los matices de la letra, aunque no renuncia por completo al estilo fresco y lleno de humor que le caracterizaba. De hecho, publicó una de las canciones más orales de su carrera ("Teología/Ideología") que narra con una gran ironía la transformación de Adán desde el paraíso hasta el marxismo.

El estilo poético se afianzó con el disco "Eskeintza" de 1991. Hay canciones, como "Habanera" o "Neguaren zai", que están métricamente influidas por el bertsolarismo, pero su lenguaje está alejado de los ecos del bertsolarismo, tan visibles en los discos anteriores.

Su último disco, basado en un concierto en directo, tiene dos canciones de cada clasificación. Este disco tampoco es muy representativo, ya que solo publicó cuatro canciones nuevas y está basado en su mayoría en canciones antiguas, generalmente no orales; Lete utiliza las dos canciones más orales para aligerar la densa carga poética de sus otras canciones. La segunda parte del concierto está basada en seis traducciones de Jacques Brel, junto a canciones antiguas.

Las explicaciones que daba el poeta antes de sus canciones sustentaban la carga poética de sus canciones. Buscaba crear un puente entre emisor y receptor, acercando al artista a su público (Lete 2011, 52). Este era uno de los puntos fuertes de Lete: su capacidad oratoria. Probablemente, era consciente de que sus canciones más líricas o elevadas eran también las más distantes para el público, y sus explicaciones las hacían más accesibles ya que las contextualizaba. Lete necesitaba ser comprendido. Es aquí donde es palpable la intención comunicativa del autor. Tenía la conciencia de que las canciones algunas veces tienen varias oportunidades para ser recibidas y entendidas, pero otras veces fracasan si no son suficientemente claras.

Uno de los mayores logros de Lete es que algunas de sus canciones, y no precisamente las más simples, han sido popularizadas y repetidas, por lo que sus letras se han convertido en parte del "ser vasco". Según Lord (70), esta comunión es posible gracias a que cantante y oyente comparten una cosmovisión: la performance puede tener lugar en cualquier sitio, pero es esencial que el 'performador' y los oyentes compartan una visión común de la vida. 
Lord afirmaba que la tradición consiste en diversos elementos: el lugar en el orden social del cuentacuentos, el arte de componer canciones en un lenguaje especial mediante fórmulas, el contenido tradicional, patrones de historias y similares (120). Lete dominaba el patrimonio literario y cultural, y lo reformulaba para alimentar los oídos de los oyentes, componiendo canciones que parecían que ya existían. Es lo que defiende Sarali Gintsburg (2019) cuando explica que la memorización de bastas cantidades de texto con contenido tradicional pueden influir crucialmente a la hora de crear o actuar ya que activan fórmulas y estructuras compartidas en una determinada sociedad.

Además, gracias a su inquietud y la relación con la familia Lekuona, Lete pudo leer a escritores de calidad, con lo que contribuía a elevar el nivel de algunas de sus composiciones de una base popular a una culta.

\section{ANÁLISIS DE TRES CANCIONES}

Se han elegido tres canciones representativas de la oralidad del autor: "Langile baten seme", "Gizon arruntaren koplak", y "Henry Miller-i eskutitza" son tres canciones transicionales en las cuales se ha puesto la lupa sobre características mencionadas anteriormente y que por tanto se relacionan con piezas orales.

"Langile baten seme" habla sobre la lucha de clases y la nimiedad del ser, y hace un paralelismo de su persona con el pueblo vasco. Esta es su primera estrofa:

$\begin{array}{ll}\begin{array}{l}\text { Langile baten semea naiz ni, } \\ \text { aita bezala langile, }\end{array} & \begin{array}{l}\text { Yo soy hijo de un trabajador, } \\ \text { trabajador como mi padre, } \\ \text { eguneroko alegiñetan }\end{array} \\ \begin{array}{l}\text { soldata baten egile; } \\ \text { esku hutsik jaio eta }\end{array} & \text { con esfuerzo diario } \\ \text { esku hutsikan nabile, } & \text { nací con las manos vacías } \\ \text { puntakoetan iñor etzaigu } & \text { sigo con las manos vacías } \\ \text { gertatu ongile. (Lete 2006, 66) } & \text { ninguno de nuestros superiores } \\ \text { ha resultado ser bueno. }\end{array}$

En la frase "esku hutsik jaio eta esku hutsikan nabile" ("nací con las manos vacías, y con las manos vacías sigo") hay algún error que el autor comete conscientemente: convierte el verbo nabil (traducido, 'ando') en nabile con el único fin de rimar. Estas licencias son comprensibles en la literatura oral donde los autores están improvisando, pero mucho menos frecuentes en la poesía es- 
crita, ya que la persona que recoge por escrito lo recientemente creado "repara" las incorrecciones del texto como recuerda Gintsburg:

To confuse matters, we know that it was normal practice for scribes to make changes to oral texts they were putting into writing in order to 'improve' their quality: scribes would 'repair' rhyme, look for better words and even replace whole lines. (476)

El autor es plenamente consciente de que está imitando un estilo oral y no duda en conservar y redundar en estas pequeñas deformaciones del lenguaje cuando lo necesita. En la tercera estrofa utiliza la palabra "Parisan" en vez de "Parisen" (traducido, 'en París'). Este es un error que un improvisador se puede permitir por las prisas y la dificultad del performance, pero una canción escrita raramente adopta. También destaca "periodikuak" en vez de "egunkariak", que evoca que el texto fuera escrito algunas décadas antes. De hecho, Lete cuenta en el último bertso que ha ["cantado docenas de bertsos corrientes"]:

Izerdi asko ez dut isurtzen

larreko pendizan

paper artean murgildutako

zomorroa izan,

limosnatik bizi diren

umezurtz batzuen gisan,

periodikuak ez dira gutaz

mintzatzen Parisan. (Lete 2006, 66)

\author{
No sudo demasiado \\ en las cuestas del huerto \\ más bien, soy un insecto \\ inmerso en papeles \\ como los huérfanos que viven \\ de las limosnas, \\ los periódicos no hablan \\ de nosotros en París.
}

Los saltos temporales que se dan durante la canción son también mencionables. La construcción de las frases como "izerdi asko ez dut isurtzen larreko pendizan" ('no sudo mucho en las cuestas de la huerta, más bien, soy un insecto inmerso en papeles') en las que una ofrece especificaciones sobre la anterior, parece también muy oral.

El uso de fórmulas es destacable en esta canción. La segunda estrofa utiliza las palabras batean, artean, kaltean, apartean como rima. Fórmulas muy similares eran frecuentemente utilizadas por Txirrita -el bertsolari más conocido del siglo XX- en decenas de bertsos, dada su versatilidad, para dar al lector el contexto del tema que iba a abordar en sus bertsos escritos (bertso-paperak). Un ejemplo puede ser este "hamarreko handia" ('décima mayor') en el que personifica a la lengua vasca y dialoga con ella: 
Ama gaixoak erantzun zidan

hain doinu triste batean:

"Minez egon naiz joan den berrogei

eta hamabost urtean;

nire malkorik ez da lehortu

ordutik orain artean

gure hizkuntza maitagarria

sartu nahi dute lurpean

baina gurekin ibiliko da

Kantabriako partean". (Lete 2001)
La pobre madre me respondió en un tono tan triste:

"He estado enferma durante

los últimos cincuenta años

mis lágrimas no se han secado

desde entonces

quieren enterrar

nuestra querida lengua

pero seguirá con nosotras

en la zona de Cantabria".

La estrofa de Lete contiene muchas elipsis, características de la oralidad; sobre todo destaca la omisión de algunos verbos. Esto confiere al texto un dinamismo característico del bertsolarismo. Las elipsis se deben, en gran parte, a la necesidad de encajar el texto en una métrica. La primera estrofa tiene una estructura que se asemeja al tradicional zortziko bandia pero con la peculiaridad de que el quinto verso es octosílabo en vez de ser decasílabo y el último verso tiene solo seis sílabas $(10,8,10,8,8,8,10,6)$.

\section{UNOS BERTSOS NADA CORRIENTES}

De toda la obra de Lete, la canción que considero más oral es "Gizon arruntaren koplak". Esta canción relata la cotidianidad de un hombre corriente.

Kantatzera noazu bertso bat edo bi

herenegun jarriak gizon arruntari

Antonio, Lorentxo edo Joxe Mari

gauza ederra denik ez ukatu neri. (Lete, 2006: 74)
Voy a cantaros uno o dos bertsos escritos al hombre común Antonio, Lorenzo, o Jose Mari no me digáis que no es hermoso.

Sus primeras estrofas son descriptivas y relativamente coherentes, pero después se convierten en una especie de divertimento con frases inconexas, casi dadaístas. Recuerda al ejercicio en el que dos bertsolaris improvisan los bertsos alternándose versos, llamado puntuka. En esta modalidad el discurso de los bertsolaris se va intercalando. Si se ejecuta sobre un zortziko txikia, el número de versos es par, por lo que el bertsolari que termina un bertso no es el que lo ha empezado. Siendo esto así, no tienen el control de a dónde se dirigen, ya que normalmente están habituados a pensar primero e último verso de la estrofa; lo que provoca que algunos bertsos no sean coherentes. En el tercer bertso (la tercera estrofa) hay un buen ejemplo de ello: 


$\begin{array}{ll}\text { Etxetikan lanera noa } & \text { Voy despacio } \\ \text { nahiko mantso, } & \text { de casa al trabajo, } \\ \text { Realak kuatro-uno } & \text { ayer la Real perdió } \\ \text { galdu zuen atzo, } & \text { cuatro a uno, } \\ \text { kalean bi zakur ta } & \text { en la calle, dos perros } \\ \text { beste hiru atso, } & \text { y tres ancianas } \\ \text { egun on don Pepito } & \text { Buenos días, don Pepito } \\ \text { eta don Alfontso. (Lete, 2006, 74) } & \text { y don Alfonso. }\end{array}$

Es como si Lete estuviera componiendo la letra en cada paso que da, alternando la descripción del narrador con conversaciones directas.

El quinto bertso es el más surrealista e inconexo. Parece estar ojeando las noticias del periódico (probablemente ya ha llegado a la oficina). En esta estrofa es destacable que utiliza el bis, la repetición de la última frase, para añadir otra frase. Esta subraya más aún la simulada improvisación e impredecibilidad de la obra. Algunos bertsolaris pueden utilizar esta técnica cuando improvisan, aun a sabiendas de que está contra la norma establecida, ya que el público seguramente comenzará a cantar la última frase para acompañar al bertsolari y el cambio lo pillará por sorpresa. La frase, además, no tiene nada de elevado, no aporta ninguna información relevante. Suena como un comentario que hizo alguien de la oficina, o un fragmento de una crónica del periódico:

$\begin{array}{ll}\text { Gauzak gaizki dabiltza, } & \text { Las cosas andan mal, } \\ \text { hau da komeriya, } & \text { ¡vaya movida! } \\ \text { Afganistan-en gerra } & \text { una guerra en Afganistán } \\ \text { piztu zuten iya, } & \text { casi comenzaron } \\ \text { tokatzen baldin bazait } & \text { Si este año } \\ \text { aurten loteriya } & \text { me toca la lotería } \\ \text { erosi beharko det } & \text { me compraré } \\ \text { Zitroen berriya } & \text { un Citroën nuevo } \\ \text { Ondo egon omen zan } & \text { dicen que estuvo bien } \\ \text { Tolosan feria. (Lete 2006, 74) } & \text { la feria de Tolosa. }\end{array}$

El sexto y el séptimo son los bertsos que más sentido tienen. Son muy correctos en cuanto a coherencia, pero estando en un contexto tan surrealista suenan irónicos.

El octavo tiene dos partes, en las que la primera hace avanzar la historia para concluir con otros dos sinsentidos: "Asko gustatzen baitzait oiloaren salda" 
('me gusta mucho el caldo de gallina') y "Mugairen hasten baita Belateko malda" ('la cuesta de Belate comienza en Mugaire'). Y termina con la fórmula de añadir una frase donde tocaba repetición. Esta vez, aportando información con mucho sentido, ya que se despide del oyente. La importancia de este final se acentúa musicalmente, porque la versión cantada repite esta parte varias veces.

Estructuralmente, es de señalar que el tercer verso de las estrofas ("Antoniyo, Lorentxo edo Joxe Mari, aurrean papera ta atzean papera, kalean bi zakur ta beste hiru atso, batzuek loriyak ta beste batzuk miak") es a menudo una enumeración sin verbos. Ong apuntaba que el estilo oral tiende a ser acumulativo más que analítico (188-212).

\section{DISCURSO SOBRE EL SEXO}

Una de las últimas canciones que compuso Lete es "Henry Miller-i eskutitza" en la que habla sobre el sexo, a propósito del conocido y controvertido escritor.

La letra de la canción son bertsos escritos con una métrica y un estilo de escritura tradicionales aunque el tema sea, quizás, innovador. Habla sobre el sexo desde un punto de vista masculino y se ríe de sí mismo. Este conjunto de bertsos compagina algunas piezas de mucha sutileza con expresiones bastante burdas.

La parte más destacable es la función que forman el tercer y cuarto verso de cada bertso. En ellos, Lete utiliza los dos primeros versos para señalar un tema, y los dos siguientes para solucionar o explicitar la frase anterior. Estos son los primeros cuatro versos de la tercera y cuarta estrofa:

$\begin{array}{ll}\begin{array}{l}\text { Maite duenak edertasuna } \\ \text { maite duenak lilura }\end{array} & \text { Quien ame la belleza, } \\ \text { hurbiletik begira dezala } & \text { y ame la fascinación } \\ \text { Platonen liburu hura. } & \text { quea con atención } \\ \text { Zahartzaroan sentitzen du batek } & \text { En la vejez, uno siente } \\ \text { bereaz konturatuta } & \text { observándose a sí mismo } \\ \text { zeinen laburra izan den uda } & \text { qué corto ha sido el verano una vez que } \\ \text { ia neguan sartuta. (Lete 2006, 104) } & \text { se ha entrado en invierno. }\end{array}$

El bertsolari Imanol Lazkano (23) afirma que para saber si un bertsolari es bueno, hay que poner la lupa en este punto exacto. Lete, consciente o inconscientemente, refleja el modo de construir los bertsos de los viejos maestros. 


\section{CONCLUSIÓN}

En este análisis se ha podido observar cómo Lete combina dos estilos, uno predominantemente poético y alejado de la oralidad, y otro semi-oral. El poeta dominaba a la perfección ambos registros. Sus discos evolucionan de lo oral a lo no oral. Probablemente, esta evolución esté relacionada con la situación política y cultural, y también con el medio de difusión. El autor necesitaba comunicar eficientemente una idea al público y solo disponía de actuaciones en directo. Esto contribuyó a que el estilo elegido fuese más oral y directo. Años más tarde, su posición cultural, tanto como poeta como de cantante, era reconocida; y el disco se convirtió en su medio de difusión principal mediante el cual se podían escuchar las canciones una y otra vez.

Lete se beneficia de las ventajas de la escritura (autoría, repercusión...) para difundir su pensamiento, pero es consciente de que la canción es una disciplina distinta a la poesía y por ello opta, algunas veces, por un lenguaje distinto del que utiliza en sus libros. La categorización más acertada para sus canciones sería la de semi-oralidad. El autor recupera el patrimonio oral directa e indirectamente, puesto que, por una parte, la musicaliza y, por otra parte, compone usando sus bases: Conoce las técnicas y expresiones, ha memorizado muchas frases y las reinterpreta (Lekuona 173) para crear un texto totalmente nuevo, pero que recuerda al oral. Lete rumió el input tradicional para crear un output vanguardista, al igual que el juglar adaptaba su texto porque la visión del mundo de su público había cambiado.

Paradójicamente, este proceso de acercamiento deriva en que gran parte de la sociedad no sea consciente de la autoría de estas canciones. Algunas de las canciones de Lete, por ejemplo "Izarren hautsa", "Xalbadorren heriotzean II" ('En la muerte de Xalbador'), "Neguaren zai”, "Seaska kanta”, "Nafarroa, arragoa" ('Navarra, crisol') son muy conocidas, pero no suelen atribuirse a Lete, sino que suelen ser tomadas por populares. A ello se le debe sumar que muchas de las letras no son concretas ya que tuvieron que superar la censura; algunas frases de sus canciones han sido descontextualizadas y usadas como lemas, algunas veces con intenciones opuestas a cuando se escribieron. Esta pérdida de control sobre su propia obra es interesante e incluso puede ser una característica del estilo oral.

Probablemente no sea casualidad el problema que tenemos en la literatura vasca para delimitar lo oral y lo escrito, y quizás partir de esquemas de otras literaturas, en las que ambos mundos están más separados, es lo que dificulta la tarea. El bertsolarismo está sustentado sobre una tradición centenaria, 
y ha perdurado gracias a las funciones que cumplía dada la tardía irrupción de la literatura escrita y las prohibiciones posteriores. Hoy en día es una disciplina artística moderna que se alimenta tanto de lo oral como de lo escrito. La oralidad y el lenguaje escrito son modalidades que se complementan e influyen mutuamente. Los improvisadores están influenciados por lo que leen y hay poetas que reconstruyen patrones orales para crear textos escritos. Esta transicionalidad suele ser, algunas veces, criticada o menospreciada por personas que desean trazar líneas claras entre disciplinas, pero la realidad es que estos dos mundos se retroalimentan desde el inicio.

Las canciones de Lete son más accesibles para el público gracias a esta influencia. Los ecos de oralidad contribuyeron a que algunos contenidos complejos fueran asimilados más fácilmente, ya que su continente era familiar para los oyentes. La lectura contribuye a que la creación de piezas improvisadas del bertsolarismo alcancen una complejidad que décadas atrás sería inimaginable.

Podemos concluir que Lete fue un filtro por el que fluía la tradición oral. Su caudal fue, además, rescatado del olvido y puesto en valor gracias a su música y su voz. Su alma quedó empapada por siempre y toda su creación posterior se impregnó de aquel líquido, esencia vasca, y por ello sus letras calaron tan hondo en la sociedad vasca, ya que parecía que existían desde siempre.

\section{OBRAS CITADAS}

Aulestia, Gorka. "Bertsolarismo: Island or Archipelago?". Voicing the Moment: Improvised Oral Poetry and Basque Tradition. Eds. Samuel G. Armistead y Joseba Zulaika. Reno: University of Nevada Press, 2005. 169-94.

Barandiaran Amarika, Asier. "El campeonato de bertsolaris de Euskal Herria como institución canonizadora de las melodías, de los ejercicios y de las estrofas de bertsos". Transitional Texts: Drifting between the Oral and the Written. Eds. S. Gintsburg, J. Ford \& A. Barandiaran. Special issue of Rilce 36.4 (2020): 1477-96.

Foley, John Miles. How to Read an Oral Poem. Urbana: University of Illinois Press, 2002.

Foley, John Miles. "Basque oral poetry championship". Oral Tradition 22.2 (2007): 3-11.

Garzia, Joxerra. "History of Improvised Bertsolarismo: A Proposal". Oral Tradition 22.2 (2007): 77-115. 
Garzia, Joxerra, Jon Sarasua y Andoni Egaña. El arte del bertsolarismo. Donostia: Bertsolari Liburuak, 2001.

Gintsburg, Sarali. "It's got some meaning but I am not sure...: The role of the particle (wa)-ma in the oral and transitional poetry of the Jbala (northern Morocco) from the cognitive perspective". Pragmatics and Cognition 24.3 (2017): 474-95.

Gintsburg, Sarali. "Lost in dictation. A cognitive approach to oral poetry: Frames, scripts and 'unnecessary' words in the Jebli ayyu". Language \& Communication 64 (2019): 104-15.

Gintsburg, Sarali, y John Ford. "Living through transition: the poetic tradition of the Jbala between orality and literacy at a time of major cultural transformations". Transitional Texts: Drifting between the Oral and the Written. Eds. S. Gintsburg, J. Ford \& A. Barandiaran. Special issue of Rilce 36.4 (2020): 1434-54.

Lazkano, Imanol. Abobizarrik gabe. Andoain: Bertsozaleak, 2003.

Lekuona, Juan Mari. Ikaskuntzak Euskal Literaturaz. Donostia: Deustuko Unibertsitatea, 1998.

Lete, Xabier. "Kanta berria, erresistentzi abestia". Jakin aldizkaria 4 (1977): 16-28.

Lete, Xabier. Txirrita 1. Donostia: Elkarlanean, 2001.

Lete, Xabier. Abestitzak eta poema kantatuak. Donostia: Elkarlanean, 2006.

Lete, Xabier. Xabier Lete (auto)biografia bat. Ed. Inazio Mujika. Irun: Alberdania, 2011.

Lord, Albert Bates. The Singer Resumes the Tale. New York: Cornell UP, 1995.

Ong, Walter J. Oralidad y escritura: tecnologías de la palabra. Trad. Angélica Scherp. Ciudad de México: FCE, 2016.

Parry, Milman. "Studies in the epic technique of oral verse-making, I: Homer and Homeric style". Harvard Studies in Classical Philology 41 (1930): 73-147.

White, Linda. "Formulas in the Mind: A Preliminary Examination to Determine if Oral Formulaic Theory May Be Applied to the Basque Case". Voicing the Moment: Improvised Oral Poetry and Basque Tradition. Eds. Samuel G. Armistead y Joseba Zulaika. Reno: University of Nevada Press, 2005. 247-63.

Wray, A. Formulaic Language: Pushing the Boundaries. Oxford: Oxford UP, 2008. 